\title{
Quantifying the local structure of incommensurately modulated tetragonal tungsten bronze from STEM images
}

\author{
Stephen Funni, Matthew Cabral and Elizabeth Dickey \\ Carnegie Mellon University, United States
}

The manner in which local structure and chemistry deviate from the average crystalline unit cell has a great influence on the properties of many materials. This is particularly true for technologically important materials such as classical and relaxor ferroelectrics. These materials find applications in capacitors, computer memory, transducers and actuators. Perovskite relaxors, most notably $\mathrm{PbMn}_{1 / 3} \mathrm{Nb}_{2 / 3} \mathrm{O}_{3}$, have been widely studied, however the physical origin of the behavior is poorly understood. ${ }^{1,2}$ Tetragonal tungsten bronze (TTB) is a metal oxide structure stable over a wide range of compositions, many exhibiting FE or relaxor behavior. ${ }^{3,4}$ TTBs are, however, much more structurally complex than perovskites, commonly having modulated superlattices and incommensurate/commensurate transitions. $^{3,5}$ The TTB structure is illustrated in Figure 1a. Zhu et al. found a correlation between A-site cation size and modulation type and further linked the presence of relaxor behavior to the onset of incommensurately modulated structures with increasing A-cation radius. ${ }^{3}$ Previous studies of the TTB modulations have been either x-ray diffraction based, focusing primarily on oxygen octahedral tilting, or used electron diffraction and dark field imaging to observe the correlation length of modulated regions., 5

To study the relationship between relaxor properties and the modulated structures in TTBs, we have employed aberration-corrected scanning transmission electron microscopy (STEM) high-angular annular dark field imaging to observe short- to long-range cation disorder on a sublattice specific basis. In this presentation, we report data from $\mathrm{Ba}_{5} \mathrm{SmSn}_{3} \mathrm{Nb}_{7} \mathrm{O}_{30}(\mathrm{BSSN})$, which is a relaxor ferroelectric. ${ }^{7}$ We chose to image along the [110] zone axis because both the modulation wave vector and the predicted A2 site cation displacements are perpendicular to this direction, allowing straightforward observation. Figure 1a shows this projection. To allow long-range correlations to be accurately measured from the data, a nonlinear drift correction code is applied to series of images taken at orthogonal scan rotations. ${ }^{8}$ Series are also captured of a silicon standard at identical imaging conditions and used to correct scan vector distortions via an affine transformation. ${ }^{9}$

We address the quantification challenges presented by the structural complexity of TTBs (including closely spaced atomic columns and 5 distinct cation column types in the [110] projection) by developing an algorithm for simultaneously locating and indexing all atom columns in a lattice image. The method uses a reference lattice generated from a unit cell structural projection which is scaled and oriented to the image using the FFT. Next, the image is segmented by applying the watershed method, which creates a mask for each atomic column. Reference lattice points are matched to their corresponding mask, inherently indexing the atomic columns. The final masks are then applied to the original image and the isolated columns are fit with 2D Gaussians to determine their location. The reference lattice is then refined by least squares minimization of the distance between the reference lattice and the fitted column positions. For BSSN, this refinement is performed on only the A1 sublattice due to its minimal displacements, which are not measurably affected by the modulation. Since the algorithm automatically determines a unique mask for each atomic column, few, if any, parameter adjustments are required (i.e. the user does not need to find an optimal thresholding value for each image). This approach is applicable to a wide range of 
structures and imaging modes. Furthermore, the use of a reference lattice enables straightforward displacement measurements. Displacement maps for three BSSN sublattices are shown in Figure 2. Note that while the A1 and B2 sublattice columns exhibit very little structural disorder, large variances in the A 21 columns are measured. Moreover, A2 1 columns show varying displacements that are consistent with the modulation vector type measured from electron diffraction: $q=\alpha\left(a^{*}+b^{*}\right)+1 / 2 c^{*}$.

To quantify the structural modulation in BSSN, we introduce the 2D pair correlation function (PCF) as a real-space crystallography method and show that it is useful for quantifying correlations in atomic displacements. Because both orientation and distance information is retained, 2D PCFs provide richer crystallographic data about the sample and are easier to interpret than 1D radial PCFs. Our 2D PCFs are based on atomic column position data and consist of distinct peaks due the crystalline structure. We quantify peak shapes by calculating the eccentricity from second order central moments. Small or large eccentricities are indicative of correlated or anti-correlated displacements. Applying this analysis to BSSN, we demonstrate that the 2D partial PCFs clearly show the presence or absence of modulations and other correlated displacements, as shown in Figure 1b. In particular, the 2D partial PCFs show that the incommensurate modulation type predicted is present on the A2 1 sublattice, but not on the other cation sublattices. Finally, we show that 2D PCFs can be used to quantify the nature of displacements, such as the periodicity of the structural modulation in BSSN and that this agrees closely with that measured by diffraction (Figure 1c).

This material is based upon work supported by the National Science Foundation, as part of the center for Dielectrics and Piezoelectrics under Grant Nos. IIP-1841453 and IIP-1841466.

This work was performed in part at the Analytical Instrumentation Facility (AIF) at North Carolina State University, which is supported by the State Of North Carolina and the National Science Foundation (award number ECCS-2025064). The AIF is a member of the North Carolina Research Triangle Nanotechnology Network (RTNN), a site in the National Nanotechnology Coordinated Infrastructure (NNCI). 


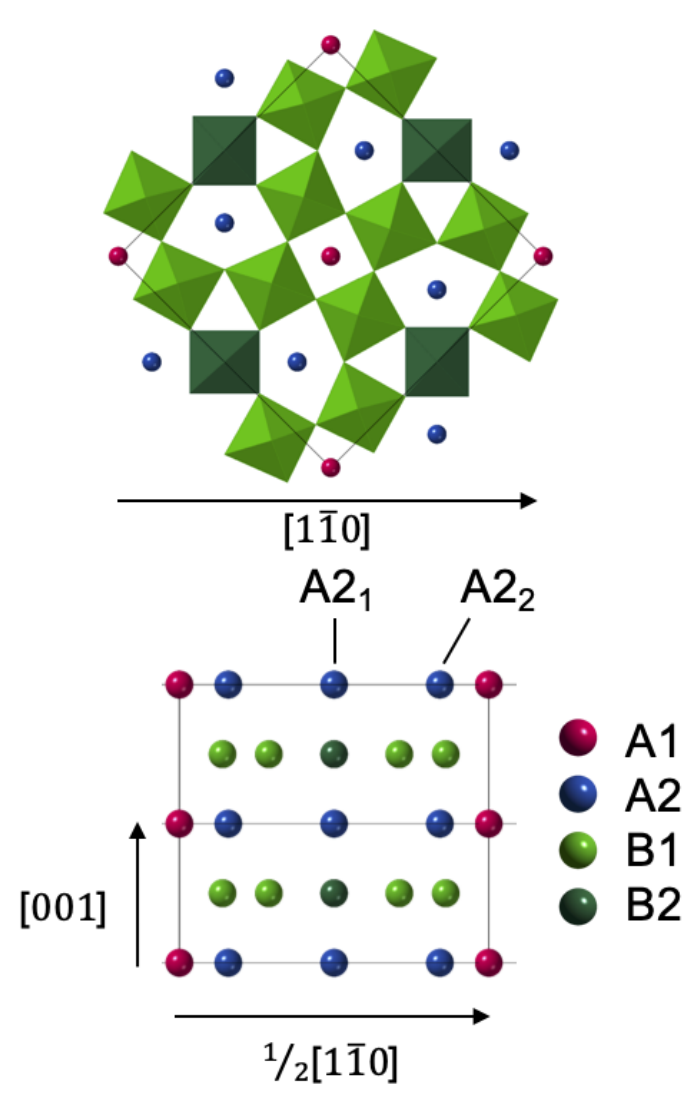

Experimental Partial Pair Correlation Functions

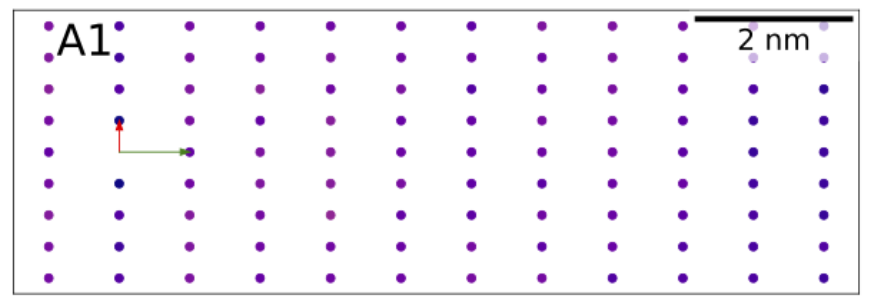
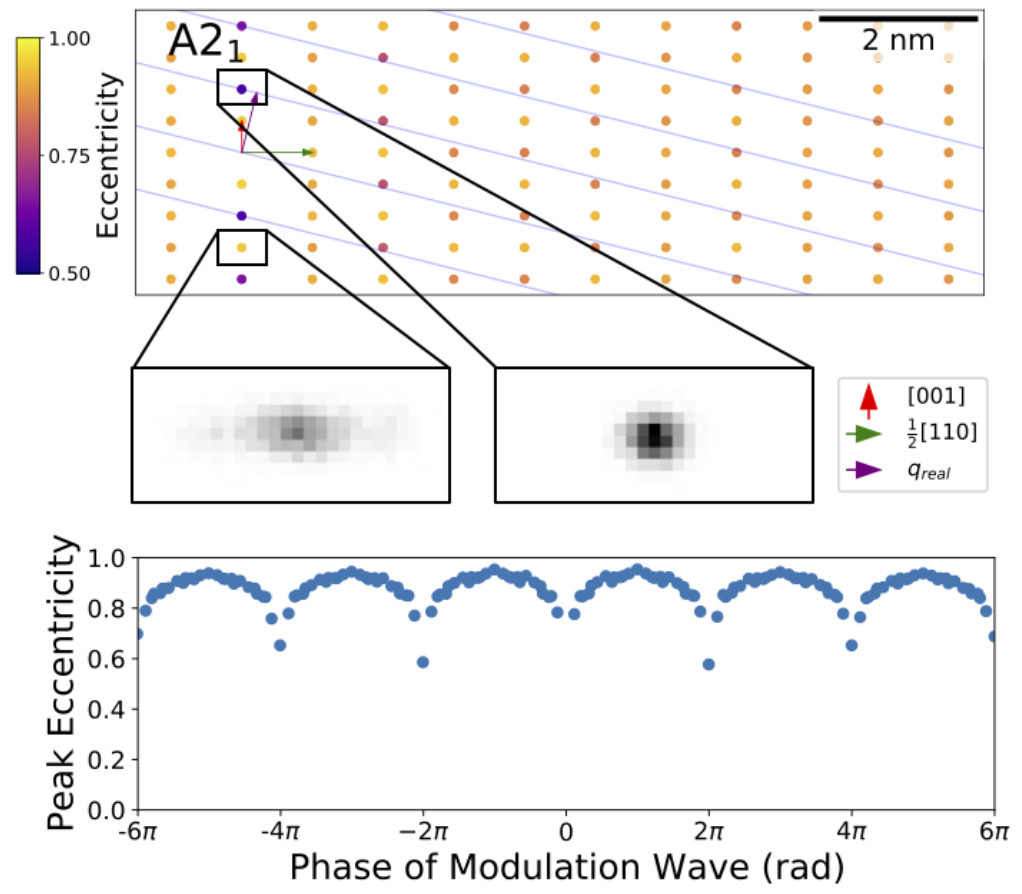

Figure 1. Figure 1: a) Structural model of the ideal TTB unit cell. b) 2D partial PCFs with no correlated displacements observed on the A1 sublattice, but a clear periodic correlated/anti-correlated displacement pattern on the A2 sublattice (as measured from the A21 atomic columns). c) Eccentricity of the A21 partial $\mathrm{PCF}$ peaks as a function of position with respect to the phase of the modulation wave.
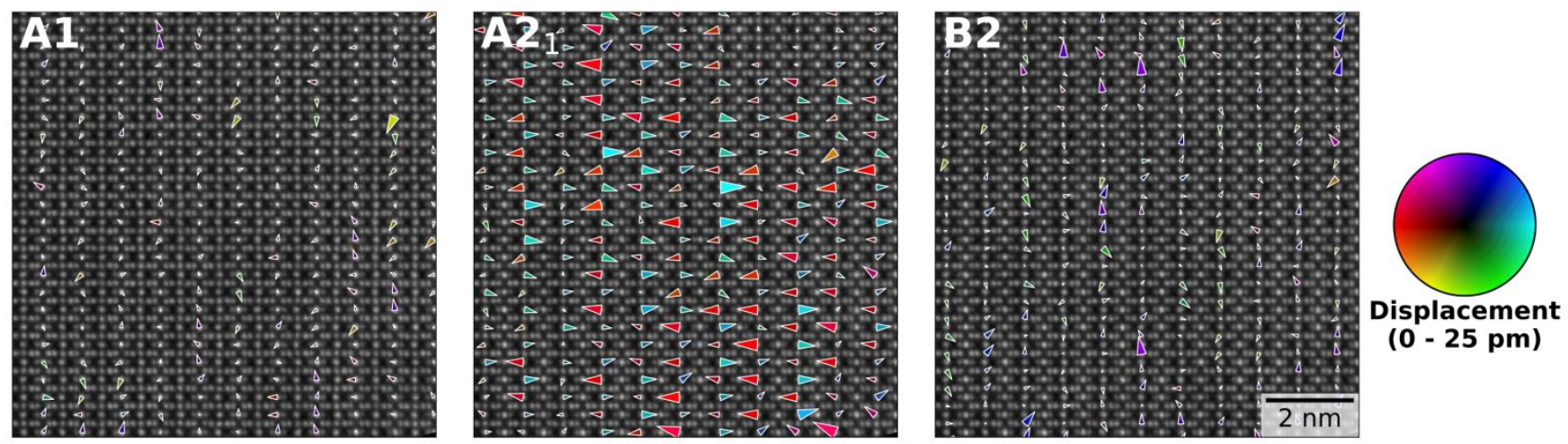

Figure 2. Figure 2: Displacement vector plots for three of the cation sublattices along the [110] zone axis. 


\section{References}

1. R. A. Cowley, S. N. Gvasaliya, S. G. Lushnikov, B. Roessli, and G. M. Rotaru: Adv. Phys. 60, 229 (2011).

2. A. A. Bokov and Z. G. Ye: Recent progress in relaxor ferroelectrics with perovskite structure. $J$. Mater. Sci. 41(1), 31 (2006).

3. X. Zhu, M. Fu, M. C. Stennett, P. M. Vilarinho, I. Levin, C. A. Randall, J. Gardner, F. D. Morrison, and I. M. Reaney: A Crystal-chemical framework for relaxor versus normal ferroelectric behavior in tetragonal tungsten bronzes. Chem. Mater. 27(9), 3250 (2015).

4. P. B. Jamieson, S. C. Abrahams, and J. L. Bernstein: Ferroelectric tungsten bronze-type crystal structures. II. Barium sodium niobate $\mathrm{Ba}(4+\mathrm{x}) \mathrm{Na}(2-2 x) \mathrm{Nb} 10030$. J. Chem. Phys. 50(10), 4352 (1969).

5. T. Woike, V. Petricek, M. Dused, N. K. Hansen, P. Fertey, C. Lecomte, A. Arakcheeva, G. Chapuis, M. Imlau, and R. Pankrath: The modulated structure of Ba 0.39 Sr 0.61 Nb 2 O 6. I. Harmonic solution. Acta Crystallogr. Sect. B59, 28 (2003).

6. L. A. Bursill, A. ) Peng, and J. U. Lin: Incommensurate Superstructures and Phase Transition of Strontium Barium Niobate (SBN). Acta Cryst 43, 49 (1987).

7. Z. J. Yang, W. Bin Feng, X. Q. Liu, X. L. Zhu, and X. M. Chen: Ba4R2Sn4Nb6O30 (R = La, Nd, $\mathrm{Sm}$ ) lead-free relaxors with filled tungsten bronze structure. J. Am. Ceram. Soc. 102(8), 4721 (2019).

8. C. Ophus, J. Ciston, and C. T. Nelson: Correcting nonlinear drift distortion of scanning probe and scanning transmission electron microscopies from image pairs with orthogonal scan directions. Ultramicroscopy 162, 1 (2016).

9. J. H. Dycus, J. S. Harris, X. Sang, C. M. Fancher, S. D. Findlay, A. A. Oni, T. T. E. Chan, C. C. Koch, J. L. Jones, L. J. Allen, D. L. Irving, and J. M. LeBeau: Accurate Nanoscale Crystallography in Real-Space Using Scanning Transmission Electron Microscopy. Microsc. Microanal. 21(4), 946 (2015). 